\title{
Coaching and Education for Diabetes Distress (CEDD): Protocol for a Randomized Controlled Trial
}

Charles C Chima ${ }^{1,2}$, MBBS, DrPH, MSc; Jason L Salemi ${ }^{2}$, PhD, MPH; Mohamad A Sidani ${ }^{2}$, MD, MS; Roger J Zoorob $^{2}$, MD, MPH

$\overline{1}$ Department of Population Health Science, John D Bower School of Population Health, University of Mississippi Medical Center, Jackson, MS, United States

${ }^{2}$ Department of Family and Community Medicine, Baylor College of Medicine, Houston, TX, United States

\section{Corresponding Author:}

Charles C Chima, MBBS, DrPH, MSc

Department of Population Health Science

John D Bower School of Population Health

University of Mississippi Medical Center

2500 North State Street

Jackson, MS, 39216

United States

Phone: 16018159544

Email: cchima@umc.edu

\begin{abstract}
Background: Diabetes distress (DD), a type of psychological distress specific to people with diabetes, is strongly associated with difficulties in performing self-care and inability to meet glycemic targets. Despite increased recognition of the need to manage DD, interventions that are both feasible and effective for reducing DD in routine care settings are not yet known. A pilot study showed that health coaching (HC) has some efficacy in addressing DD, but no adequately powered study has implemented a pragmatic research design capable of assessing the real-world effectiveness of HC in reducing DD.

Objective: The aim of this study is to describe the rationale and design of an ongoing clinical trial, Coaching and Education for Diabetes Distress trial, that seeks to assess whether HC effectively reduces DD among primary care patients with diabetes and whether $\mathrm{HC}$ is more effective than an educational program targeting DD.

Methods: The 2-arm randomized controlled trial is taking place at an academic family medicine practice in Houston, Texas. Both arms will receive usual care, which includes education about DD. In addition, the intervention arm will receive $8 \mathrm{HC}$ sessions over a 5-month period. The primary outcome measure is reduction in DD over a 6-month period. Additional outcome measures include changes in hemoglobin $\mathrm{A}_{1 \mathrm{c}}$ and self-care practices (medication-taking, dietary, and physical activity behaviors).

Results: As of March 2019, screening and recruitment are ongoing, and the results are expected by July 2020.

Conclusions: $\mathrm{HC}$ is feasible in primary care and has been successfully applied to improving chronic disease self-management and outcomes. This study will provide evidence as to whether it has significant value in addressing important unmet psychological and behavioral needs of patients with diabetes.
\end{abstract}

Trial Registration: ClinicalTrials.gov NCT03617146; https://clinicaltrials.gov/ct2/show/NCT03617146 (Archived by WebCite at http://www.webcitation.org/76Va37dbO)

International Registered Report Identifier (IRRID)： DERR1-10.2196/12166

(JMIR Res Protoc 2019;8(4):e12166) doi: 10.2196/12166

\section{KEYWORDS}

diabetes mellitus, type 2; diabetes distress; coaching; health coaching; counselling; self care; behavioral medicine; health psychology; health education; primary care 


\section{Introduction}

Diabetes distress (DD) is a negative emotional reaction to diagnosis of diabetes and concerns about treatment demands, risk of complications, and inadequate support [1]. DD, along with major depressive disorder (MDD) and other depressive symptomatology, constitutes significant emotional and psychological burden to people with diabetes [1,2]. Among these distinct conditions, DD is most strongly associated with difficulties in performing self-care and inability to meet glycemic targets [2-4], and it is more common than MDD [5], leading to calls for DD management to become an essential component of diabetes care $[1,6]$.

A review by Sturt et al [7] identified 6 approaches to address types of DD that have been studied; namely, psychological (eg, psychotherapy), educational (purely informational), educational with some behavioral component, psychoeducational (education plus a psychological intervention targeting motivation or affect), medications and devices, and care management or case management [7]. Of these, psychoeducational approaches were the only approaches found to significantly reduce DD compared with controls. Similarly, a subsequent review [8] found that DD showed improvement following interventions that target both emotion (motivational strategies) and cognition (enlightenment or education).

Psychoeducational interventions that target emotion and cognition are a broad category that includes strategies such as health coaching (HC) and psychotherapy plus education; therefore, it is necessary to identify emotion-cognition interventions that are both feasible and effective for reducing $\mathrm{DD}$ in routine care settings. $\mathrm{HC}$ is an evolving profession that helps individuals achieve sustainable behavioral change through a growth-promoting relationship that elicits autonomous motivation and improves knowledge, self-efficacy, and self-regulation [9]. HC is particularly important to diabetes care as it has the potential to address both DD and self-care.

The American Diabetes Association (ADA) recommends that providers "routinely monitor people with diabetes for DD, particularly when treatment targets are not met..." [10]. However, ADA's recommendations for dealing with DD (ie, education, referral to a behavioral health provider, or referral to a mental health specialist) are varied and confusing [10]. On the other hand, the Canadian Diabetes Association [11] recommends an approach that is consistent with $\mathrm{HC}$ as defined by Olsen [12] and Wolever et al [13]. Even though some interventions have incorporated elements of coaching $[7,8]$, only 1 study has explicitly sought to address DD as a primary outcome using HC [14]. This study found HC to be effective in addressing DD, but the finding has limited generalizability because of methodological limitations such as a small sample size and restriction to a single gender [14]. A recent trial that combined diabetes self-management education with some elements of $\mathrm{HC}$ and additional support was successful in addressing DD, but the intervention was too intensive to be feasible and scalable in routine primary care [15]. We proposed to conduct a randomized controlled trial (RCT) to fill the void in the available evidence on the real-world effectiveness of $\mathrm{HC}$ on DD. The primary aims of the study are to assess whether education alone is sufficient to address DD and whether HC has additional benefits in addressing DD beyond the effects of education. As coaching services are not typically reimbursed by insurance, cost has been a barrier to greater integration of $\mathrm{HC}$ in chronic illness care [16]. Therefore, one of the secondary aims of the study is to assess the willingness of patients with DD to pay for HC. The other secondary aim is to find out whether $\mathrm{HC}$ has additional impact on diabetes self-care and hemoglobin $\mathrm{A}_{1 \mathrm{c}}\left(\mathrm{Hb}_{1 \mathrm{c}}\right)$, beyond any effects observed in the group that receives only an educational program targeting DD.

\section{Methods}

\section{Study Design and Setting}

A 2-arm, parallel RCT is being conducted among adults with diabetes who are not meeting glycemic targets (defined as $\mathrm{HbA}_{1 \mathrm{c}}$ $\geq 8.0$ ), at an academic family medicine clinic in Houston, Texas. The overarching goal of the study is to use a pragmatic approach to assess the effectiveness of $\mathrm{HC}$ in addressing DD in real-world primary care settings, in which most diabetes care is coordinated and monitored. Therefore, participants randomized to the control group will receive the current standard of care for patients not meeting glycemic targets at the study clinic as summarized in Textbox 1 .

Both arms of the study (control and intervention) will receive the standard of care detailed in Textbox 1. In addition, the intervention arm will receive $8 \mathrm{HC}$ sessions to address DD. The $\mathrm{HC}$ sessions will be held biweekly in the first 3 months (6 sessions), followed by monthly sessions in the fourth and fifth month. 
Textbox 1. Standard of care for diabetes at the study site.

- Quarterly follow-up visits during which patients receive ongoing, comprehensive assessment and treatment. When patients with diabetes whose $\mathrm{HbA}_{1 \mathrm{c}}$ is 8.0 or above exceed a 4-month period without a visit, they are contacted to schedule a follow-up appointment.

- Pharmacologic treatment of diabetes and comorbidities

- Behavioral management-diabetes self-management education and support (DSMES), that covers lifestyle modification counseling (nutrition and physical activity), medication-taking behaviors, foot care, how to monitor blood glucose (especially for those on insulin), and how to administer insulin if on insulin. This ongoing DSMES is provided through different means as follows:

- Directly by primary care providers

- Via health education handouts in the form of printed materials and via an electronic patient portal

- By referral to a registered dietitian located at the clinic

- Referral to an ophthalmology clinic for annual eye check-up

- Psychological management - as part of a newly developed quality improvement project, and in line with the 2018 American Diabetes Association's Standards of Medical Care in Diabetes [10], the clinic has implemented psychological screening (depression and diabetes distress) for patients not meeting their glycemic targets (defined as $\mathrm{HbA}_{1 \mathrm{c}} \geq 8.0$ ). The plan of action is as follows:

- $\quad$ Patients who screen positive for depression (using the Patient Health Questionnaire) [17] will be referred to colocated mental health providers.

- A diabetes distress (DD)-specific education program has been developed for patients with DD and is being piloted through this study (Multimedia Appendix 1). This education program is oriented around the 4 domains of DD-regimen distress, emotional burden, interpersonal distress, and physician distress. Patients are provided feedback on DD screening results and are advised on ways to deal with the areas of greatest distress. Screening for DD is done using the 17-item DD scale (DDS17) [18].

\section{Description of the Coaching Intervention for Patients With Diabetes Distress}

\section{Coaching Approach}

Even as $\mathrm{HC}$ is becoming more common in primary care, there is a wide and varied understanding of what constitutes HC [12]. For the purposes of this study, the authors use the term $\mathrm{HC}$ to refer to an intervention with a goal of facilitating health behavior change, conducted by a health care professional (eg, registered nurse, registered dietitian, medical assistant, clinical psychologist) who has additional training and certification as a health coach. Thus, interventions carried out by peers or lay people will not meet this standard. The authors align with the conceptualization of $\mathrm{HC}$ as articulated in the reviews by Olsen [12] and Wolever et al [13] and reflected in the following definition:
A patient-centered approach wherein patients at least partially determine their goals, use self-discovery or active learning processes together with content education to work toward their goals, and self-monitor behaviors to increase accountability, all within the context of an interpersonal relationship with a coach. The coach is a healthcare professional trained in behavior change theory, motivational strategies, and communication techniques, which are used to assist patients to develop intrinsic motivation and obtain skills to create sustainable change for improved health and well-being. [12]

Interventions for alleviating DD need to address 2 issues: the first is helping the patient express his or her feelings about diabetes, and the second is to work with the patient to figure out what can be done to meet the patient's needs [19]. Coaching techniques suitable for achieving the first objective include building a growth-promoting relationship with the client, establishing trust and rapport through the application of 3 core coaching skills, namely mindful listening, open-ended inquiry, and perceptive reflections [20], and helping clients handle negative emotions through an empathy protocol such as nonviolent communication [21]. To address the second objective of meeting the patient's needs, the coach will help participants elicit self-motivation, build self-efficacy, and journey through a process of change to reach their desired goals, relying on techniques such as exploring and amplifying the best in the client through appreciative inquiry [22] and building self-efficacy through motivational interviewing [23].

The abovementioned techniques for addressing the 2 issues in DD provide the overall framework that the coach will work with to help participants address DD and overcome the underlying challenges contributing to their distress. Among these techniques, motivational interviewing is probably the best described and standardized, and it has been demonstrated to be effective in many settings [24-28].

A true HC approach recognizes that each patient is unique, focuses on meeting the needs of the patient, and emphasizes relationship development between the coach and client $[12,13]$. As each participant's needs and values will be unique, the coach will tailor the intervention to the needs of each participant while staying within the overall coaching framework described above. This approach of not restricting the intervention to a rigid protocol is in keeping with the understanding that flexibility within the overall coaching paradigm is key to successful coach-client collaboration [12,13].

All coaching sessions will be delivered over the phone (ie, telecoaching) to minimize inconvenience, transportation time, and cost demands to participants. Telecoaching has been previously shown to be highly acceptable, low-cost, and 
effective in increasing autonomy and self-efficacy among adult patients with diabetes [29].

\section{Participant Assessment}

Before the first coaching session, the participant will fill out the diabetes distress scale (DDS) [18] and a subset of the Summary of Diabetes Self-Care Activities questionnaire [30] focused on medication-taking, dietary, and physical activity behaviors. Only patients with significant DD (DDS score $\geq 2.0$ ) will be included in this study. The health coach will review the DDS report to obtain information on the specific type of distress (eg, regimen distress) and the primary sources of distress, that is, the specific things about diabetes management that are upsetting to that particular person (eg, not feeling motivated to keep up with diabetes self-management; see Table 1).

\section{First Coaching Session}

At the first coaching session, building on the findings from the participant assessment, the coach will probe further to identify possible targets for the coaching intervention (Table 1). The targets define the specific goals related to DD or the underlying stressors that the participant would like to resolve or achieve at the end of the intervention. As necessary, the coach might also explore participant's priorities, confidence, and readiness to change concerning different aspects of diabetes self-management. The coach then explores the participant's willingness to embark on the coaching journey, discusses the findings from the assessment, and works with the participant to identify possible coaching targets (Table 1). The participant prioritizes the coaching targets and creates goals that he or she will like to achieve and a 3-month action plan.

\section{Subsequent Coaching Sessions}

Participants' progress will be reviewed at subsequent coaching sessions. Most coaching sessions focus on a specific topic, helping clients navigate and overcome emerging challenges on their change journey [9]. As necessary, the coach will tap into her or his coaching toolbox to help elevate the participant's energy, brainstorm strategies for problem solving, develop solutions, meet challenges, and set and agree on subsequent goals [9].

For purposes of quality control, the health coach will take notes on client interactions and coaching techniques used for review with the investigators. This allows ongoing feedback to ensure appropriateness of the intervention.

\section{Training of Intervention Staff}

$\mathrm{HC}$ will be provided by a registered dietitian trained and certified as a Health and Wellness Coach by one of the "Transition Programs" approved by the International Consortium for Health and Wellness Coaching [31]. The International Consortium for Health and Wellness Coaching is an organization working to standardize HC practice in the United States and across the world. DD-specific education (Multimedia Appendix 1) for participants in both arms of the study will be provided by designated medical assistants in the clinic who were trained to provide this education in a consistent manner (Textbox 1).

\section{Study Population, Inclusion, and Exclusion Criteria}

The target population is adult patients with diabetes and DD at the study clinic. Textbox 2 summarizes the inclusion and exclusion criteria.

Table 1. Identifying the focus of the coaching intervention from the type and sources of diabetes distress using the diabetes distress scale.

\begin{tabular}{|c|c|c|}
\hline $\mathrm{DDS}^{\mathrm{a}}$ subscale & Questions (from DDS) & Possible coaching targets \\
\hline Regimen distress & $\begin{array}{l}\text { Not feeling confident in my day-to-day ability to manage diabetes; Feeling that I } \\
\text { am not testing my blood sugars frequently enough; Feeling that I am often failing } \\
\text { with my diabetes routine; Feeling that I am not sticking closely enough to a good } \\
\text { meal plan; Not feeling motivated to keep up my diabetes self-management }\end{array}$ & $\begin{array}{l}\text { Perception of low self-efficacy for diabetes } \\
\text { self-care; Perception of low motivation for } \\
\text { diabetes self-care; Perception of lack of } \\
\text { success in following diabetes self-care plan }\end{array}$ \\
\hline Emotional burden & $\begin{array}{l}\text { Feeling that diabetes is taking up too much of my mental and physical energy every } \\
\text { day; Feeling angry, scared, depressed, or a mixture of these feelings when I think } \\
\text { about living with diabetes; Feeling that I will end up with serious long-term com- } \\
\text { plications, no matter what I do; Feeling that diabetes controls my life; Feeling } \\
\text { overwhelmed by the demands of living with diabetes }\end{array}$ & $\begin{array}{l}\text { Low level of perceived ability to influence } \\
\text { outcomes; Low level of perceived ability to } \\
\text { manage emotions }\end{array}$ \\
\hline $\begin{array}{l}\text { Interpersonal dis- } \\
\text { tress }\end{array}$ & $\begin{array}{l}\text { Feeling that friends or family are not supportive enough of self-care efforts; Feeling } \\
\text { that friends or family do not appreciate how difficult living with diabetes can be; } \\
\text { Feeling that friends or family do not give me the emotional support that I would } \\
\text { like }\end{array}$ & $\begin{array}{l}\text { Perception of low levels of social support } \\
\text { for dealing with diabetes }\end{array}$ \\
\hline
\end{tabular}

${ }^{\mathrm{a}} \mathrm{DDS}$ : diabetes distress scale. 
Textbox 2. Inclusion and exclusion criteria for the Coaching and Education for Diabetes Distress trial.

Inclusion criteria

- Has had a diagnosis of type 2 diabetes for at least 6 months

- $\quad$ Aged 18 to 75 years

- Most recent hemoglobin $\mathrm{A}_{1 \mathrm{c}}$ taken within 30 days was 8.0 or above

- At least a moderate diabetes distress, operationalized as a mean score of 2.0 or more on the 17 -item diabetes distress scale [32]

Exclusion criteria

- Moderately-severe to severe depression: Patient health questionnaire-9 score 15 or above [17]

- Other severe mental health disorder (eg, Alzheimer's or schizophrenia)

- Current pregnancy

- Severe diabetes complications or functional deficits (eg, kidney failure requiring dialysis, amputation, or blindness)

\section{Study Arm Assignment}

Figure 1 outlines the screening, recruitment, and randomization process for the study. A random sorting randomization algorithm will be implemented using PASS software version 15.0.3 (NCSS LLC, Kaysville, UT) to generate a randomization list that will dictate assignment of patients into either the intervention or control (usual care) arm. To ensure the algorithm results in the desired group sample sizes, the program search is conducted by creating a randomization list using the user-specified randomization algorithm and then looking at the final sample sizes. If the sample sizes do not match the target sample sizes for all groups, then the list is discarded and the algorithm is restarted. This process continues until a list with the exact sample sizes is found.

Figure 1. Screening, recruitment, and randomization process for Coaching and Education for Diabetes Distress trial.

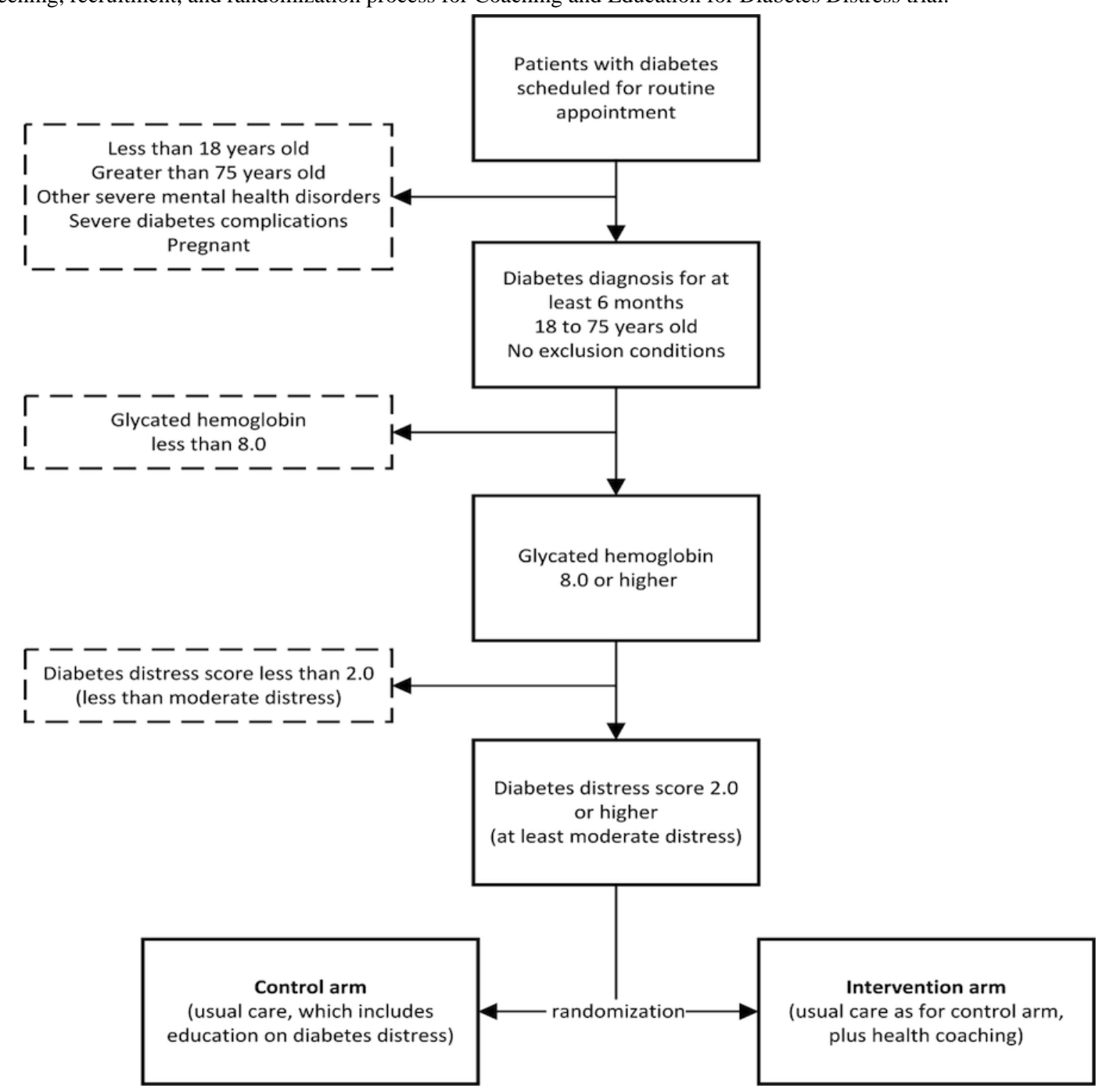




\section{Sample Size Determination}

The study requires a total sample of 156 participants, 39 in the intervention arm and 117 in the control arm. Calculations were based on the following assumptions: a power of $80 \%$, alpha (type I error rate) of 5\%, an effect size (difference in mean change in DD between the 2 groups) of 0.5 , which is considered both clinically significant and at least of moderate magnitude [7], a DD SD of 0.89 and 0.81 , respectively, at baseline and at follow-up [15], a correlation $(\rho)$ of 0.5 between a pair of observations made longitudinally on the same subject, and accounting for a possible attrition rate of $20 \%$. It has been shown that when research costs vary among treatments, it is economically efficient to randomize more participants to the cheaper arm without compromising validity [33]. Therefore, to achieve adequate statistical power within fiscal constraints, more participants will be recruited in the control arm than in the intervention arm (at a ratio of $3: 1$ ). On the basis of these assumptions and the stated sample size, this study achieves $80 \%$ statistical power to detect differences in the mean change in DD of 0.5 between the 2 groups, but it has more than $90 \%$ power to detect differences in mean changes in DD of 0.6 or higher (Figure 2).

\section{Measures and Data Collection}

The variables that will be measured and the timeframe for data collection are summarized in Table 2. The primary outcome measure will be short-term (6 months) changes in DD score. Secondary outcome measures of interest are willingness-to-pay (WTP) for and satisfaction with $\mathrm{HC}$, as well as changes in $\mathrm{HbA}_{1 \mathrm{c}}$ and diabetes self-care practices, specifically dietary intake, physical activity, and medication-taking behaviors.

$\mathrm{DD}$, self-care behaviors, and $\mathrm{HbA}_{1 \mathrm{c}}$ will be assessed at 3 measurement periods, at baseline (randomization), and at the third and sixth month postrandomization. DD will be assessed using the previously validated 17-item DDS [18]. $\mathrm{HbA}_{1 \mathrm{c}}$ will be tested at every 3-month follow-up visit as part of usual care for patients not meeting glucose targets. Self-care practices (diet, physical activity, and medication-taking behaviors) will be assessed with the respective subscales of the Summary of Diabetes Self-Care Activities questionnaire [30]. Satisfaction with $\mathrm{HC}$ will be assessed for the intervention arm at the end of the study (sixth month) through self-administered surveys, including Likert scale-type questions and open-ended questions. WTP for HC will be assessed through an indirect survey approach, which has been shown to be preferable because of higher internal and external validity compared with other methods [34]. In such indirect approaches, participants are confronted with different attribute combinations of various products or services (eg, $\mathrm{HC}$ vs alternative interventions) with assigned prices, and they choose the most preferred scenarios. Specifically, we will conduct a discrete choice experiment to assess WTP.

Figure 2. Statistical power of Coaching and Education for Diabetes Distress trial to detect effect sizes from 0.4 to 1.0.

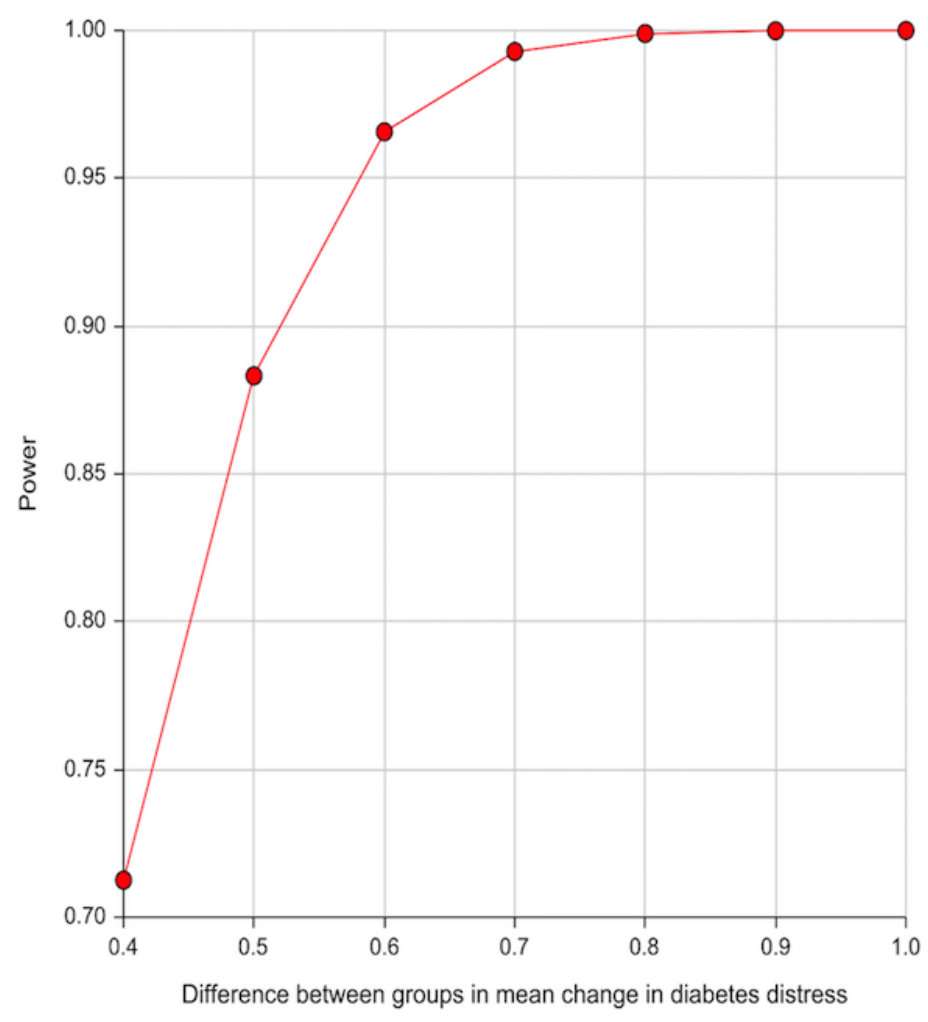


Table 2. Measures and time frame for the Coaching and Education for Diabetes Distress trial.

\begin{tabular}{|c|c|c|c|}
\hline Measure & Baseline & 3 months from baseline & 6 months from baseline \\
\hline \multicolumn{4}{|l|}{ Primary outcome measure } \\
\hline Diabetes distress score [18] & $X$ & $\mathrm{X}$ & $\mathrm{X}$ \\
\hline \multicolumn{4}{|l|}{ Secondary outcome measures } \\
\hline Hemoglobin $\mathrm{A}_{1 \mathrm{c}}$ & $\mathrm{X}$ & $\mathrm{X}$ & $\mathrm{X}$ \\
\hline $\begin{array}{l}\text { Self-care behaviors (Medication-taking, dietary, and physical activity behaviors) } \\
\text { using the Summary of Diabetes Self-Care Activities questionnaire [30] }\end{array}$ & $X$ & $\mathrm{X}$ & $\mathrm{X}$ \\
\hline Willingness to pay for health coaching & $\mathrm{X}$ & $-{ }^{\mathrm{a}}$ & $\mathrm{X}$ \\
\hline Satisfaction with health coaching (intervention arm only) & - & - & $\mathrm{X}$ \\
\hline
\end{tabular}

${ }^{\mathrm{a}}$ Not applicable.

\section{Data Analysis}

The primary analysis will use repeated measures analysis of variance to compare mean change in $\mathrm{DD}, \mathrm{HbA}_{1 \mathrm{c}}$, and self-care scores between baseline and months 3 and 6 for the 2 study arms. Satisfaction with HC will be reported as the proportion of respondents agreeing with different grades of a Likert scale. The open-ended questions on the HC satisfaction questionnaire will be analyzed qualitatively using thematic analysis. On the basis of the choices that the participants make in the discrete choice experiment, WTP for HC will be estimated by logistic regression models.

\section{Ethical Clearance}

Patients eligible for the study will be offered the opportunity to enroll; however, the decision to enroll will be completely up to the patient. Patients who decline to enroll will continue to receive the usual standard of care; therefore, they will be informed that their decision to opt out will not affect their care in any way. An informed consent will be obtained from all patients who decide to participate. Ethical approval for the study has been obtained from the Institutional Review Board of the Baylor College of Medicine, Houston, Texas.

\section{Results}

As of March 2019, screening and recruitment for the Coaching and Education for Diabetes Distress (CEDD) trial are ongoing and will continue through December 2019. The results will be expected by July 2020.

\section{Discussion}

Over 30 million Americans (9.4\% of the US population) were living with diabetes mellitus in 2015 [35]. With an estimated expenditure of US $\$ 101.4$ billion in 2013, the United States spent more on managing diabetes than any other condition [36]. Furthermore, diabetes is the seventh leading cause of death [35] and the leading cause of adult-onset blindness, kidney failure, and nontraumatic limb amputations in adults [37]. Therefore, interventions that demonstrate real-world effectiveness in improving diabetes management have the potential to improve quality of life, reduce mortality, and decrease costs to patients, their families, and society at large.

The need to address psychological and behavioral factors as essential components of comprehensive diabetes care is increasingly being recognized. However, an effective and easily scalable intervention for DD, the most common psychological barrier to diabetes management, is not yet known. Whittemore et al [15] found that $\mathrm{HC}$ resulted in better self-care and reduced $\mathrm{DD}$, but it had no significant effect on $\mathrm{HbA}_{1 \mathrm{c}}$; however, their study was limited to a small sample of women who previously voluntarily participated in diabetes education at an outpatient diabetes education center. Such women might have more intrinsic motivation than a general population of primary care patients, and they certainly might have different results from a population which includes men; therefore, the findings may not be generalizable to a general population of primary care patients. This paper reports on the design of an ongoing RCT of $\mathrm{HC}$ and education for DD (CEDD trial) that overcomes the methodological limitations of previous studies of coaching-related interventions for DD. The findings of this study will provide targeted evidence as to whether $\mathrm{HC}$ has significant value in addressing important unmet psychological and behavioral needs of patients with diabetes.

As coaching services are typically not covered by medical insurance plans at the point of care, cost has been a barrier to greater integration of $\mathrm{HC}$ in chronic illness care [16]. Therefore, assuming that this study finds that $\mathrm{HC}$ is superior to distress-specific education in addressing DD, issues of payment might constitute a barrier to scalability. By also assessing the degree to which patients with diabetes would be willing to pay for $\mathrm{HC}$ out of pocket, this study could pave the way for mainstreaming of $\mathrm{HC}$ in diabetes care. These findings would have far-reaching implications for diabetes management in routine primary care settings.

\section{Acknowledgments}

This clinical trial is supported by the Harnisch Research Project Grant of the Institute of Coaching. The content of this publication is solely the responsibility of the authors and the grantor had no influence on it. 


\section{Conflicts of Interest}

None declared.

\section{Multimedia Appendix 1}

Diabetes Distress Education Script.

[PDF File (Adobe PDF File), 90KB-Multimedia Appendix 1]

\section{References}

1. Gonzalez JS, Fisher L, Polonsky WH. Depression in diabetes: have we been missing something important? Diabetes Care 2011 Jan;34(1):236-239 [FREE Full text] [doi: 10.2337/dc10-1970] [Medline: 21193621]

2. Fisher L, Skaff MM, Mullan JT, Arean P, Mohr D, Masharani U, et al. Clinical depression versus distress among patients with type 2 diabetes: not just a question of semantics. Diabetes Care 2007 Mar;30(3):542-548. [doi: 10.2337/dc06-1614] [Medline: 17327318]

3. Fisher L, Glasgow RE, Strycker LA. The relationship between diabetes distress and clinical depression with glycemic control among patients with type 2 diabetes. Diabetes Care 2010 May;33(5):1034-1036 [FREE Full text] [doi: 10.2337/dc09-2175] [Medline: 20150291]

4. Fisher L, Mullan JT, Arean P, Glasgow RE, Hessler D, Masharani U. Diabetes distress but not clinical depression or depressive symptoms is associated with glycemic control in both cross-sectional and longitudinal analyses. Diabetes Care 2010 Jan;33(1):23-28 [FREE Full text] [doi: 10.2337/dc09-1238] [Medline: 19837786]

5. Fisher L, Skaff MM, Mullan JT, Arean P, Glasgow R, Masharani U. A longitudinal study of affective and anxiety disorders, depressive affect and diabetes distress in adults with Type 2 diabetes. Diabet Med 2008 Sep;25(9):1096-1101 [FREE Full text] [doi: 10.1111/j.1464-5491.2008.02533.x] [Medline: 19183314$]$

6. Zagarins SE, Allen NA, Garb JL, Welch G. Improvement in glycemic control following a diabetes education intervention is associated with change in diabetes distress but not change in depressive symptoms. J Behav Med 2012 Jun;35(3):299-304. [doi: 10.1007/s10865-011-9359-z] [Medline: 21691844]

7. Sturt J, Dennick K, Hessler D, Hunter BM, Oliver J, Fisher L. Effective interventions for reducing diabetes distress: systematic review and meta-analysis. International Diabetes Nursing 2015 Jul 06;12(2):40-55. [doi: 10.1179/2057332415Y.0000000004]

8. Chew BH, Vos RC, Metzendorf M, Scholten RJ, Rutten GE. Psychological interventions for diabetes-related distress in adults with type 2 diabetes mellitus. Cochrane Database Syst Rev 2017 Dec 27;9:CD011469. [doi:

10.1002/14651858.CD011469.pub2] [Medline: 28954185]

9. Moore M, Tschannen-Moran B, Jackson E. Coaching Psychology Manual. Philadelphia: Lippincott Williams \& Wilkins; 2015:8.

10. American Diabetes Association. 2018. Lifestyle Management: Standards of Medical Care in Diabetes-2018 URL: http:/ /care.diabetesjournals.org/content/diacare/41/Supplement 1/S38.full.pdf [accessed 2019-03-05] [WebCite Cache ID 76eSicLAw]

11. Canadian Diabetes Association. Using the Diabetes Distress Scale URL: http://guidelines.diabetes.ca/docs/resources/ using-the-diabetes-distress-scale.pdf [accessed 2018-09-09] [WebCite Cache ID 72JaC7AD1]

12. Olsen JM. Health coaching: a concept analysis. Nurs Forum 2014;49(1):18-29. [doi: 10.1111/nuf.12042] [Medline: 24456550]

13. Wolever RQ, Simmons LA, Sforzo GA, Dill D, Kaye M, Bechard EM, et al. A systematic review of the literature on health and wellness coaching: defining a key behavioral intervention in healthcare. Glob Adv Health Med 2013 Jul;2(4):38-57 [FREE Full text] [doi: 10.7453/gahmj.2013.042] [Medline: 24416684]

14. Whittemore R, Melkus GD, Sullivan A, Grey M. A nurse-coaching intervention for women with type 2 diabetes. Diabetes Educ 2004;30(5):795-804. [Medline: 15510531]

15. Fisher L, Hessler D, Glasgow RE, Arean PA, Masharani U, Naranjo D, et al. REDEEM: a pragmatic trial to reduce diabetes distress. Diabetes Care 2013 Sep;36(9):2551-2558 [FREE Full text] [doi: 10.2337/dc12-2493] [Medline: 23735726]

16. Wagner TH, Willard-Grace R, Chen E, Bodenheimer T, Thom DH. Costs for a health coaching intervention for chronic care management. Am J Manag Care 2016 Dec 01;22(4):e141-e146 [FREE Full text] [Medline: 27143350]

17. Kroenke K, Spitzer RL, Williams JB. The PHQ-9: validity of a brief depression severity measure. J Gen Intern Med 2001 Sep;16(9):606-613 [FREE Full text] [Medline: 11556941]

18. Polonsky WH, Fisher L, Earles J, Dudl RJ, Lees J, Mullan J, et al. Assessing psychosocial distress in diabetes: development of the diabetes distress scale. Diabetes Care 2005 Mar;28(3):626-631. [Medline: 15735199]

19. Snouffer E. International Diabetes Federation. 2016. Diabetes distress: a real and normal part of diabetes URL: https://www. idf.org/component/attachments/attachments.html?id=589\&task=download [accessed 2018-09-10] [WebCite Cache ID 72KegZ3B5]

20. Moore M, Tschannen-Moran B, Jackson E. Coaching Psychology Manual. Philadelphia: Lippincott Williams \& Wilkins; 2015:34-38. 
21. Moore M, Tschannen-Moran B, Jackson E. Coaching Psychology Manual. Philadelphia: Lippincott Williams \& Wilkins; 2015:58-62.

22. Moore M, Tschannen-Moran B, Jackson E. Coaching Psychology Manual. Philadelphia: Lippincott Williams \& Wilkins; 2015:65-74.

23. Moore M, Tschannen-Moran B, Jackson E. Coaching Psychology Manual. Philadelphia: Lippincott Williams \& Wilkins; 2015:77-87.

24. Butterworth S, Linden A, McClay W. Health coaching as an intervention in health management programs. Dis Manage Health Outcomes 2007;15(5):299-307 [FREE Full text] [doi: 10.2165/00115677-200715050-00004]

25. Emmons KM, Rollnick S. Motivational interviewing in health care settings. Opportunities and limitations. Am J Prev Med $2001 \mathrm{Jan} ; 20(1): 68-74$. [Medline: 11137778]

26. Linden A, Butterworth SW, Prochaska JO. Motivational interviewing-based health coaching as a chronic care intervention. J Eval Clin Pract 2010 Feb;16(1):166-174. [doi: 10.1111/j.1365-2753.2009.01300.x] [Medline: 20367828]

27. Burke BL, Arkowitz H, Menchola M. The efficacy of motivational interviewing: a meta-analysis of controlled clinical trials. J Consult Clin Psychol 2003 Oct;71(5):843-861. [doi: 10.1037/0022-006X.71.5.843] [Medline: 14516234]

28. Knight KM, McGowan L, Dickens C, Bundy C. A systematic review of motivational interviewing in physical health care settings. Br J Health Psychol 2006 May;11(Pt 2):319-332. [doi: 10.1348/135910705X52516] [Medline: 16643702]

29. McGloin H, Timmins F, Coates V, Boore J. Exploring the potential of telephone health and wellness coaching intervention for supporting behaviour change in adults with diabetes. J Diabetes Nurs 2015;19(10):394-400 [FREE Full text]

30. Toobert DJ, Hampson SE, Glasgow RE. The summary of diabetes self-care activities measure: results from 7 studies and a revised scale. Diabetes Care 2000 Jul;23(7):943-950 [FREE Full text] [Medline: 10895844]

31. International Consortium for Health \& Wellness Coaching. Approved Transition Programs URL: https://ichwc.org/ approved-programs/ [accessed 2018-09-10] [WebCite Cache ID 72JbszElh]

32. Fisher L, Hessler DM, Polonsky WH, Mullan J. When is diabetes distress clinically meaningful?: establishing cut points for the Diabetes Distress Scale. Diabetes Care 2012 Feb;35(2):259-264 [FREE Full text] [doi: 10.2337/dc11-1572] [Medline: 22228744]

33. Torgerson D, Campbell M. Unequal randomisation can improve the economic efficiency of clinical trials. J Health Serv Res Policy 1997 Apr;2(2):81-85. [Medline: 10180369]

34. Breidert C, Hahsler M, Reutterer T. A review of methods for measuring willingness-to-pay. Innov Mark 2006;2(4):8-32 [FREE Full text]

35. Centers for Disease Control and Prevention. National Diabetes Statistics Report, 2017 URL: https://www.cdc.gov/diabetes/ pdfs/data/statistics/national-diabetes-statistics-report.pdf [accessed 2018-09-09] [WebCite Cache ID 72JdDMkXi]

36. Dieleman JL, Baral R, Birger M, Bui AL, Bulchis A, Chapin A, et al. US spending on personal health care and public health, 1996-2013. J Am Med Assoc 2016 Dec 27;316(24):2627-2646 [FREE Full text] [doi: 10.1001/jama.2016.16885] [Medline: 28027366]

37. Centers for Disease Control and Prevention. Atlanta, GA; 2017. Diabetes Quick Facts URL: https://www.cdc.gov/diabetes/ basics/quick-facts.html [accessed 2018-09-09] [WebCite Cache ID 72JdOTypk]

\section{Abbreviations}

ADA: American Diabetes Association

CEDD: Coaching and Education for Diabetes Distress

DD: diabetes distress

DDS: diabetes distress scale

HbA $_{1 \mathbf{c}}$ : hemoglobin $\mathrm{A}_{1 \mathrm{c}}$ (glycated hemoglobin)

HC: health coaching

MDD: major depressive disorder

RCT: randomized controlled trial

WTP: willingness to pay

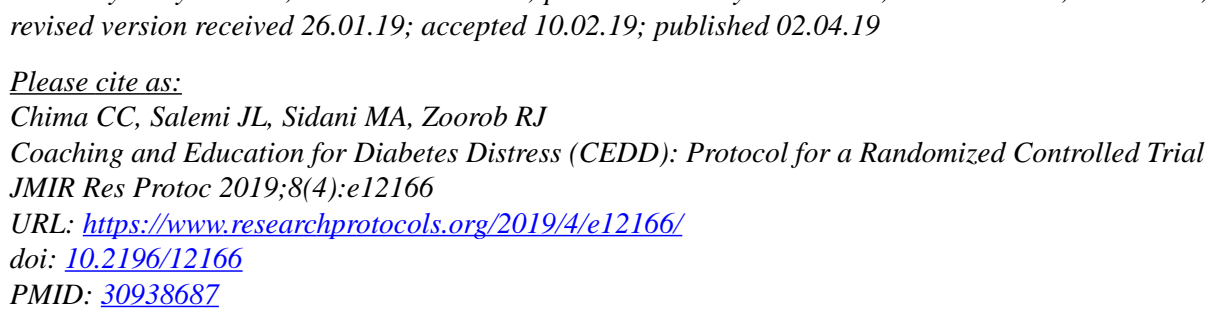


(C)Charles C Chima, Jason L Salemi, Mohamad A Sidani, Roger J Zoorob. Originally published in JMIR Research Protocols (http://www.researchprotocols.org), 02.04.2019. This is an open-access article distributed under the terms of the Creative Commons Attribution License (https://creativecommons.org/licenses/by/4.0/), which permits unrestricted use, distribution, and reproduction in any medium, provided the original work, first published in JMIR Research Protocols, is properly cited. The complete bibliographic information, a link to the original publication on http://www.researchprotocols.org, as well as this copyright and license information must be included. 